\title{
Modelos DE ELECCIÓN DisCRETA APLICADOS AL TRANSPORTE AÉREO
}

\author{
Claudia Helena Muñoz-Hoyos ${ }^{1}$
}

${ }^{1}$ Estudiante de Doctorado en Ingeniería Civil, Universidad Nacional de Colombia, Medellín, Colombia

Correo electrónico:chmunozh@unal.edu.co

Recibido: 20 de noviembre del 2013. Aprobado: 12 de marzo del 2014.

Cómo citar este artículo: C. H. Muñoz-Hoyos, "Modelos de elección discreta aplicados al transporte aéreo". Ingeniería Solidaria, vol. 10, n. 17, 179-182, en-dic. 2014. doi: http://dx.doi.org/10.16925/in.v10i17.879

Resumen. Este artículo presenta los resultados de una encuesta de preferencias declaradas en un experimento de elección discreta, diseñado para obtener una función de utilidad para las dos terminales aeroportuarias que sirven al área metropolitana del Valle de Aburrá en el transporte aéreo de pasajeros. El estudio investiga acerca del comportamiento en la elección del Aeropuerto José María Córdoba (JMC) y Enrique Olaya Herrera ( $\mathrm{EOH}$ ) entre destinos comunes. Cada aeropuerto fue representado en términos del costo del tiquete, costo de llegada a la terminal aeroportuaria y tiempo de viaje al aeropuerto y obtuvieron modelos logit multinomial para cada destino evaluado Medellín-Bogotá y Medellín-Cali.

Palabras clave: modelo de elección discreta, aeropuertos, transporte aéreo, preferencias declaradas, función de utilidad.

\section{Discrete Choice Models Applied to Air Transport}

Abstract. This article presents the results of a survey of expressed preferences in a discrete choice experiment, designed to obtain a utility function for airport terminals serving the metropolitan area of the Valle de Aburrá in air passenger transport. The study examined behavior in choosing between the José María Córdoba (JMC) and Enrique Olaya Herrera (вон) airports for the same destinations. Each was represented in terms of ticket cost, cost of arrival to the airport terminal and travel time to the airport and multinomial logit models were obtained for each Medellín-Bogotá and Medellín-Cali destination evaluated.

Keywords: discrete choice model, airports, air transport, expressed preferences, utility function.

\section{MODELOS DE ESCOLHA DISCRETA APLICADOS AO TRANSPORTE AÉREO}

Resumo. Este artigo apresenta os resultados de uma pesquisa de opinião declarada numa experiência de escolha discreta, desenhado para obter uma função de utilidade para os dois terminais aeroportuários que servem à área metropolitana do Valle de Aburrá no transporte aéreo de passageiros. Este estudo investiga sobre o comportamento na escolha do Aeroporto José María Córdoba (JMC) e Enrique Olaya Herrera (ЕОH) entre destinos comuns. Cada aeroporto foi representado em termos do custo da passagem, custo da chegada ao aeroporto e tempo de viagem ao aeroporto, e obtiveram modelos logit multinomial para destino avaliado Medellín-Bogotá e Medellín-Cali.

Palavras-chave: modelo de escolha discreta, aeroportos, transporte aéreo, preferências declaradas, função de utilidade. 


\section{Introducción 1}

El transporte aéreo es uno de los servicios más importantes de la economía colombiana. En primer lugar, debido a la topografía del país que dificulta la operación de otro tipo de transporte, facilita la integración y la conectividad; en segundo lugar, permite la conexión con la economía mundial, hecho que resulta imperativo dada la dinámica de la globalización en el mundo.

En este sentido, el transporte aéreo es considerado un servicio público esencial, lo cual pone de manifiesto su papel como un factor vital para el desarrollo económico nacional. Un usuario del modo aéreo tiene por ejemplo la opción de viajar de Medellín a Bogotá por cualquiera de los dos aeropuertos que sirven a la ciudad de Medellín, teniendo en cuenta que desde ambas terminales se ofrecen variedad de tarifas, además del tiempo y el costo que el usuario experimenta al llegar a cada una de ellas.

Para evaluar la elección de una terminal aeroportuaria, Muñoz desarrolla una investigación para modelar su demanda, mediante la calibración de un modelo de elección discreta con base en la aplicación de una encuesta de preferencias declaradas (PD), fundamentada en juicios declarados por individuos acerca de cómo actuarían frente a la situación de viajar a un destino determinado por cualquiera de los dos aeropuertos disponibles en el Valle de Aburrá [1].

Es así que se generan modelos logit multinomial y logit mixto; por medio de estos modelos se puede analizar el comportamiento de los individuos ante las dos alternativas existentes.

\section{Metodología}

En el campo de la modelación del transporte, se han utilizado los modelos de elección discreta para abordar las elecciones modales, tratando de representar el comportamiento de un individuo que debe realizar una elección dentro de un conjunto de alternativas (por ejemplo, viajar por el JMC o por el ЕОH) para realizar un desplazamiento.

En los modelos de elección discreta, también denominados modelos de respuesta cualitativa, se relaciona la probabilidad condicional de una elección con una serie de factores explicativos que recogen las características de

1 Este artículo se deriva de una ponencia presentada en el I Congreso Nacional de Vías, realizado en noviembre de 2013, en Medellín (Antioquia), Colombia. los individuos que toman las decisiones, así como los atributos de las alternativas, y permiten calcular la probabilidad de que un individuo con determinadas características haga una elección dada.

La técnica de preferencias declaradas aplicada en la investigación se basa en juicios hechos por individuos acerca de cómo actuarían frente a diferentes situaciones hipotéticas que le son presentadas y que deben ser lo más aproximadas a la realidad [2]. Estas técnicas utilizan diseños experimentales para construir las alternativas hipotéticas presentadas a los encuestados. A partir de allí, se obtienen datos que permiten estimar funciones de utilidad con respecto a las alternativas presentes en el experimento.

Las encuestas de preferencias declararas fueron realizadas en las salas de espera de los aeropuertos Enrique Olaya Herrera y José María Córdova en las rutas Medellín-Bogotá y Medellín-Cali.

\subsection{Modelos de elección discreta}

Para evaluar la demanda de transporte, además de los estudios tradicionales basados en encuestas origen y destino, se utilizan modelos de elección discreta basados en la teoría de la utilidad aleatoria, que permiten abordar empíricamente el problema de modelación en el marco de elecciones discretas. Para su calibración, es necesario obtener datos mediante la aplicación de encuestas específicas, que pueden ser de preferencias reveladas (PR) o de preferencias declaradas (PD) [2].

En el campo de la modelación del transporte, se han utilizado los modelos de elección discreta para abordar las elecciones modales, tratando de representar el comportamiento de un individuo que debe realizar una elección dentro de un conjunto de alternativas (por ejemplo, viajar por el JMC o por el ЕОН) con el fin de realizar un desplazamiento.

El problema de maximización de la utilidad tiene en cuenta, además de la alternativa elegida y del grupo de alternativas disponibles, los bienes de consumo continuo; tal como se muestra en (1) y sujeto a restricciones de presupuesto y tiempo, como se aprecia en (2) y (3).

Aplicando este problema al caso del transporte, se involucra la variable tiempo como: el tiempo de viaje y el tiempo restante disponible para el desarrollo de otras actividades.

$$
\max _{k}\left\langle\max _{x} U\left(x, q_{k}, t v_{k}, t_{\tau}\right\rangle / k \in K\right.
$$

U: $\quad$ función de utilidad 
$x$ : bienes de consumo continuo

$q_{k}$ : características de la opción $k$ (alternativa elegida)

$t v_{k}: \quad$ tiempo de viaje

$t_{\tau}: \quad$ tiempo destinado a los distintos usos $\tau$ dados al tiempo, una vez elegida la alternativa $k$.

$K$ : $\quad$ conjunto de alternativas disponibles

Sujeto a:

$$
\begin{aligned}
& p x+c k=I \text { Restricción de presupuesto } \\
& t v_{k}+\sum T t \tau k=24 \text { Restricción de tiempo }
\end{aligned}
$$

En la práctica, el problema de maximización se resuelve y da origen a una función de utilidad condicionada en cada alternativa. Al calcular la utilidad relativa entre las diferentes alternativas, lo cual es aquello que realmente evalúa cada usuario, el aporte del ingreso a dicha utilidad desaparece tal como se muestra a continuación, en la función de utilidad que suele postularse. De esta manera, el usuario elige la alternativa $k$ que le genera mayor utilidad como se indica en (4).

$$
V_{k}=\alpha c_{k}+\sum \beta_{l} q_{k l}, \forall k
$$

a y $\beta$ : parámetros que representan el impacto del atributo cualitativo $q i$ sobre el nivel de utilidad.

La anterior $(V k)$ es la utilidad conocida por el individuo, diferenciada de aquella conocida por el modelador. Para equilibrar ambas utilidades, en (5) se introduce a la conocida por el modelador un término de error aditivo, que refleja el desconocimiento de este.

$$
U_{k}=\alpha c_{k}+\sum_{l} \beta_{l} q_{k l}+\varepsilon_{k}=V_{k}+\varepsilon_{k}, \forall k
$$

\section{Estimación de los modelos por trayecto}

El modelo pendiente de estimación tomará en cuenta las utilidades asignadas por los usuarios a los dos aeropuertos disponibles. Dicha utilidad en términos generales tendrá la estructura básica mostrada en (6).

$$
U_{I}=\beta+\theta_{\text {costo tiquete }} C T_{i}+\theta_{\text {costo desplazamiento }} C D_{i}+\theta_{\text {tiempo viaje }} T V_{i}
$$

La formulación para el modelo logit multinomial (MNL) en el destino Bogotá se presenta en (7) y (8).

$$
U_{I M C}=-0,369-0,0000141 C T_{J M C}-0,0000157 C D_{J M C}-0,0167 T V_{E O H}+0,514 S E X+0,871 E D+0,485 N A V A G(7)
$$




$$
U_{\text {ЕOH }}=-0,00000141 C T_{E O H}-0,0000157 C D_{E O H}-0,0167 T_{E O H}-1,05 M T A G+0,256 T R+0,458 T A G
$$

La formulación de la función de utilidad del modelo MNL en el trayecto Medellín-Cali se describe en (9) y (10).

$$
\begin{gathered}
U_{J M C}=0,994-0,0000120 C T_{J M C}-0,0000186 C D_{J M C}-0,0205 T V_{J M C}+0,705 E S A G \\
U_{\text {EOH }}=-0,0000120-C T_{E O H}-0,0000186 C D_{E O H}-0,0205 T V_{E O H}-0,437 T R+0,888 \text { MOTAG }+1,26 \text { NAVAG }
\end{gathered}
$$

\section{Conclusiones}

A partir de la información recolectada en la encuesta de preferencias declaradas, se formularon una serie de modelos tipo logit multinomial. En estos modelos se evidencian los factores principales que impactan al individuo al momento de tomar la decisión de por cuál aeropuerto viajar.

La encuesta se aplicó a 200 individuos, y se obtuvo información socioeconómica de estos, la cual es útil para tratar de explicar por qué eligieron una terminal aeroportuaria para iniciar su viaje. Esta información se complementó con una encuesta de preferencias declaradas, en la que los individuos debían seleccionar la alternativa que les fuera más atractiva dentro de un grupo de dos posibles (viajar por el JMC o por ЕOH), en un juego de 9 casos por encuestado.

En el modelo encontrado para el trayecto Medellín-Bogotá, las variables que influyen en la elección del aeropuerto son el costo del tiquete (Ст), el costo de llegar a cada terminal (CD), el tiempo de viaje al aeropuerto
(TV), el sexo, la edad, el tipo de aeronaves en los que se prefiere viajar, el medio de transporte para llegar al aeropuerto, el tiempo de reserva del vuelo y el motivo del viaje.

Para el modelo encontrado para el trayecto Medellín-Cali, las variables influyentes en la elección del aeropuerto son el costo del tiquete (СT), el costo de llegar a cada terminal (CD), el tiempo de viaje al aeropuerto (TV), el estrato socioeconómico, el tiempo de reserva, el motivo del viaje y el tipo de aeronaves en los que se prefiere viajar.

\section{Referencias}

[1] C. Muñoz, "Modelación de la elección de la terminal aeroportuaria entre dos aeropuertos cercanos". Tesis de Maestría de la Universidad Nacional de Colombia, sede Medellín, 2012.

[2] J. de D. Ortúzar, Modelos econométricos de elección discreta. Ediciones Universidad Católica de Chile, 2000. 